N. Aoki

Nagoya Math. J.

Vol. 90 (1983), 119-135

\title{
TOPOLOGICAL STABILITY OF SOLENOIDAL AUTOMORPHISMS
}

\author{
NOBUO AOKI
}

\section{§ 0. Introduction}

In [10] A. Morimoto proved that every topologically stable homeomorphism of a compact manifold $M$ has the pseudo-orbit tracing property in the case $\operatorname{dim}(M) \geqslant 2$. Further, in studying relation between the topological stability and other stability of diffeomorphisms, he showed the following

THEOREM A. Let $\boldsymbol{R}^{r}$ be the $r$-dimensional vector group and $\varphi$ be a group automorphism of $\boldsymbol{R}^{r}$. Then the following conditions are mutually equivalent;

(i) $\varphi$ is hyperbolic,

(ii) $\varphi$ is expansive,

(iii) $\varphi$ is structually stable,

(iv) $\varphi$ has the pseudo-orbit tracing property,

(v) $\varphi$ is topologically stable.

The statement further is true for toral automorphisms.

We know (cf. see §1) that every toral automorphism is contained in the class of solenoidal automorphisms. Thus it will be natural to ask what kind of solenoidal automorphisms have the pseudo-orbit tracing property. Our aim is to investigate this problem by using results in [2] and A. Morimoto [9, 10, 11].

\section{§1. A main result and preparatory lemmas}

Let $f: X \longleftrightarrow$ be a homeomorphism of a compact metric space $(X, d)$. We denote by $\mathscr{H}(X)$ the group of all homeomorphisms of $X$. Then $\mathscr{H}(X)$ becomes a complete topological group with the topology given by the metric $d(f, g)=\max \left\{d(f(x), g(x)), d\left(f^{-1}(x), g^{-1}(x)\right): x \in X\right\}(f, g \in \mathscr{H}(X))$. We

Received December 16, 1981. 
call $f$ to be topologically stable iff for every $\varepsilon>0$ there is $\delta>0$ with the property that for every $g \in \mathscr{H}(X)$ with $d(f, g)<\delta$ there is a continuous map $h: X \longleftrightarrow$ such that

$$
\text { i) } h \circ g=f \circ h, \quad \text { ii) } d(h(x), x)<\varepsilon(x \in X) \text {. }
$$

A sequence of points $\left\{x_{i}\right\}_{i \in(a, b)}(-\infty \leqslant a<b \leqslant+\infty)$ is called a $\delta$-pseudoorbit of $f$ iff $d\left(f\left(x_{i}\right), x_{i+1}\right)<\delta$. Given $\varepsilon>0$, a $\delta$-pseudo-orbit $\left\{x_{i}\right\}$ is called to be $\varepsilon$-traced by a point $y \in X$ iff $d\left(f^{i}(y), x_{i}\right)<\varepsilon$ for every $i \in(a, b)$. We call $f$ to have the pseudo-orbit tracing property (abbrev. P.O.T.P.) iff for every $\varepsilon>0$ there is $\delta>0$ such that every $\delta$-pseudo-orbit of $f$ can be $\varepsilon$ traced by some point $y \in X$. We denote by $\operatorname{Orb}^{\delta}(f)$ the set of all (finite or infinite) $\delta$-pseudo-orbits of $f$ and by $\operatorname{Tr}^{\varepsilon}\left(\left\{x_{i}\right\}, f\right)=\operatorname{Tr}^{\varepsilon}\left(\left\{x_{i}\right\}\right)$ the set of all $y \in X$ such that $\left\{x_{i}\right\}$ is $\varepsilon$-traced by $y$. We call $(X, f)$ to have weak specification iff for every $\varepsilon>0$ there is $M(\varepsilon)>0$ such that for every $k \geqslant 1$ and $k$ points $x_{1}, \cdots, x_{k} \in X$ and for every set of integers $a_{1} \leqslant b_{1}<a_{2} \leqslant b_{2} \ldots$ $<a_{k} \leqslant b_{k}$ with $a_{i+1}-b_{i} \geqslant M(\varepsilon)(1 \leqslant i \leqslant k-1)$ there is $x \in X$ with $d\left(f^{n}(x)\right.$, $\left.f^{n}\left(x_{i}\right)\right)<\varepsilon\left(a_{i} \leqslant n \leqslant b_{i}, 1 \leqslant i \leqslant k\right)$.

We say that $X$ is solenoidal iff $X$ is a compact connected finite-dimensional abelian group. Every finite-dimensional torus is clearly solenoidal.

Hereafter $X$ will be an $r$-dimensional solenoidal group and $\sigma$ will be an automorphism of $X$. Our main result is the following

Theorem 1. The following (A) and (B) are equivalent;

(A) $(X, \sigma)$ is topologically stable,

(B) $(X, \sigma)$ has the P.O.T.P..

Further there exist solenoidal automorphisms with P.O.T.P. such that one of the following conditions holds:

(C) $(X, \sigma)$ is not expansive,

(D) $(X, \alpha)$ is not densely periodic.

The second statement of Theorem 1 will follow from Remark 1 mentioned below.

Denote by $(G, \gamma)$ the dual of $(X, \sigma)((\gamma g)(x)=g(\sigma x), g \in G$ and $x \in X)$. Let $\bar{G}$ be a minimal divisible extension of $G$ (p. 168, [7]). Since $G$ is torsion free, $\bar{G}$ is so and $\operatorname{rank}(\bar{G})=\operatorname{rank}(G)=r<\infty$ (p. 34, [7]). It is well known that $\gamma$ induces an automorphism $\bar{\gamma}$ of $\bar{G}$. We shall write $\gamma=$ $\bar{\gamma}$ for the sake of simplicity.

Let $Q\left[x, x^{-1}\right]$ be the ring of polynomials in $x$ and $x^{-1}$ with coefficients 
in $\boldsymbol{Q}$ (the notation $\boldsymbol{Q}$ means the rational field). Since $\bar{G}$ is divisible and torsion free, for every $\bar{f} \in \bar{G}$ and every natural number $n$ there is a unique $\bar{g} \in \bar{G}$ such that $n \bar{g}=\bar{f}$. So we consider $\bar{g}$ to be $(1 / n) \bar{f}$ and $\boldsymbol{Q}\left[x, x^{-1}\right]$ to act on $\bar{G}$ by $\left(\sum_{j=-m}^{n} b_{j} x^{j}\right) \bar{g}=\sum_{j=-m}^{n} b_{j} \gamma^{j} \bar{g}\left(b_{j} \in \boldsymbol{Q}\right.$ and $\left.\bar{g} \in \bar{G}\right)$. Then $\bar{G}$ becomes a $\boldsymbol{Q}\left[x, x^{-1}\right]$-module. Since $\boldsymbol{Q}\left[x, x^{-1}\right]$ is a principal ideal domain, it follows (cf. p. 397, [6]) that there is in $G$ a finite sequence $\left\{g_{1}, \ldots, g_{s}\right\}$ such that $\bar{G}$ splits into a direct sum $\bar{G}=\bar{G}_{g_{1}} \oplus \cdots \oplus \bar{G}_{g_{s}}$ of $\gamma$-invariant subgroups $\bar{G}_{g_{i}}$ where $\bar{G}_{g_{i}}=\left\{f \in \bar{G}: m f \in \operatorname{gp}\left\{\gamma^{j} g_{i}: j \in Z\right\}\right.$ for some $\left.m \neq 0\right\}$ for $1 \leqslant i \leqslant s$ (the notation gp $E$ means the subgroup generated by a set $E$ ). Since $r \bar{G}_{g_{i}}=\bar{G}_{g_{i}}$ for $1 \leqslant i \leqslant s$, we can find a polynomial $q_{i}(x) \in Z[x]$ with minimal degree $r_{i}$ such that $q_{i}(\gamma) g_{i}=0$ holds, so that $\Theta=\left\{g_{1}, \cdots, \gamma^{r_{1}-1} g_{1}\right.$, $\left.\cdots, g_{s}, \cdots, \gamma^{r_{s}-1} g_{s}\right\}$ is linearly independent (the notation $Z[x]$ means the ring of polynomials with integer coefficients). Hence the factor group $G / \mathrm{gp} \Theta$ is a torsion group; i.e. gp $\Theta$ is full in $G$. Numbering the elements of $\Theta$ as $\Theta=\left\{e_{1}, \cdots, e_{r}\right\}$, every $0 \neq g \in G$ is expressed as $a g=a_{1} e_{1}+\cdots+a_{r} e_{r}$ for some $a \neq 0$ and some $a_{1}, \cdots, a_{r}$ with $\left(a_{1}, \cdots, a_{r}\right) \neq(0, \cdots, 0)$. Since the existence of $\left(a_{1} / a, \cdots, a_{r} / a\right)$ is unique, we can define an into isomorphism $\varphi: G \rightarrow \boldsymbol{Q}^{r}$ by $\varphi(g)=\left(a_{1} / a, \cdots, a_{r} / a\right)$. To simplify the notations, we identify $g$ with $\left(a_{1} / a, \cdots, a_{r} / a\right)$ under $\varphi$. Then $\Theta$ is the canonical basis of $\boldsymbol{Z}^{r}$ (i.e. $\left.e_{1}=(1,0, \cdots, 0), \cdots, e_{r}=(0, \cdots, 0,1)\right)$, so that $\operatorname{gp} \Theta=\boldsymbol{Z}^{r} \subset G \subset \bar{G}=\boldsymbol{Q}^{r}$ $\subset \boldsymbol{R}^{r}$. We extend $\gamma$ on $\boldsymbol{R}^{r}$ by the natural way, and denote it by the same symbol. For $t=\left(t_{1}, \cdots, t_{r}\right) \in \boldsymbol{R}^{r}$, define $\psi(t) g=t_{1} a_{1} / a+\cdots+t_{r} a_{r} / a$ (addition $\bmod 1)$ for all $g=\left(a_{1} / a, \cdots, a_{r} / a\right) \in G$. Then we get $\psi(t) \in X$ (p. 251, [12]). In fact, $\psi: \boldsymbol{R}^{r} \rightarrow X$ is an into homomorphism. The adjoint map $\hat{\gamma}$ of $\gamma$ is defined by $\psi(\hat{\gamma} t) g=\psi(t) r g=(\sigma \psi(t)) g\left(t \in \boldsymbol{R}^{r}, g \in G\right)$. Since $\hat{\gamma}$ and $\gamma$ are isomorphic, we denote $\hat{\gamma}$ by $\gamma$ again, and say $\left(\boldsymbol{R}^{r}, \hat{\gamma}\right)$ to be the lifting system of $(X, \sigma)$.

Lemma B ((P.2(i)), [2]). Under the above notations, $\psi\left(\boldsymbol{R}^{r}\right)$ is dense in $X$. If $X$ is a torus then $\psi\left(\boldsymbol{R}^{r}\right)=X$.

Lemma C ((P.2), [2]). Let $F$ be the annihilator of gp $\Theta$ in $X$. Then (i) $F$ is totally disconnected and $\psi^{-1}\left\{\psi\left(\boldsymbol{R}^{r}\right) \cap F\right\}=Z^{r}$, (ii) $X=\psi\left(\boldsymbol{R}^{r}\right)+F$ and (iii) there is a small closed neighborhood $U$ of 0 in $\boldsymbol{R}^{r}$ such that $\psi(U)$ $\cap F=\{0\}$ and the direct product $U \times F$ is homeomorphic to $\psi(U)+F$. And $\psi(U)+F$ is a closed neighborhood of 0 in $X$ (We write $\psi(U) \oplus F$ such a neighborhood $\psi(U)+F)$.

Lemma D. Under the above notations, the followings hold; 
(i) there exist a torus $V_{1}$ and a vector group $V_{2}$ such that $\psi$ induces a 1-1 homomorphism $\psi^{*}$ from the direct product group $V_{1} \oplus V_{2}$ onto $\psi\left(\boldsymbol{R}^{r}\right)$,

(ii) $\psi\left(\boldsymbol{Z}^{r}\right)$ is a closed subgroup in $\psi\left(\boldsymbol{R}^{r}\right)$,

(iii) $\psi\left(Z^{r}\right)$ is dense in $F$.

Proof. Let $K$ be the kernel of $\psi$. Then $K \subset Z^{r}$ by Lemma C(i), so that there are a torus $V_{1}$ and a vector subgroup $V_{2}$ such that $\boldsymbol{R}^{r} / K=V_{1}$ $\oplus V_{2}$. Therefore $\psi$ induces a 1-1, onto homomorphism $\psi^{*}: V_{1} \oplus V_{2} \rightarrow \psi\left(\boldsymbol{R}^{r}\right)$ in the natural way. (i) was proved. It is clear that $\overline{\psi\left(Z^{r}\right)} \subset F$. By Lemma C (ii), $\overline{\psi\left(\boldsymbol{Z}^{r}\right)} \cap \psi\left(\boldsymbol{R}^{r}\right) \subset F \cap \psi\left(\boldsymbol{R}^{r}\right)=\psi\left(\boldsymbol{Z}^{r}\right)$ and so $\overline{\psi\left(\boldsymbol{Z}^{r}\right)} \cap \psi\left(\boldsymbol{R}^{r}\right)=\psi\left(\boldsymbol{Z}^{r}\right)$. This shows (ii). Put $B=\overline{\psi\left(Z^{r}\right)}$. Then $X / B=\left\{\left(\psi\left(\boldsymbol{R}^{r}\right)+B\right) / B\right\}+\{F / B\}$. Since $\psi\left(\boldsymbol{R}^{r}\right) / \psi\left(\boldsymbol{Z}^{r}\right)$ is a factor group of $\boldsymbol{R}^{r} / \boldsymbol{Z}^{r}$, it is a torus and so $\left(\psi\left(\boldsymbol{R}^{r}\right)+\right.$ $B) / B$ is also a torus since $\left(\psi\left(\boldsymbol{R}^{r}\right)+B\right) / B \cong \psi\left(\boldsymbol{R}^{r}\right) / \psi\left(\boldsymbol{Z}^{r}\right)$. On the other hand, $X / B$ is connected, from which we have $F=B$.

Lemma E. Let $V_{1}, V_{2}$ and $\psi^{*}$ be as in Lemma D. For $\alpha_{2}>0$ small enough, let $B\left(\alpha_{2}\right)$ be a closed neighborhood with the radius $\alpha_{2}$ of 0 in $V_{1} \oplus$ $V_{2}$. Then $B\left(\alpha_{2}\right)$ is a closed neighborhood of $\boldsymbol{R}^{r}$ and $\psi^{*}(v)=\psi(v)$ for every $v \in B\left(\alpha_{2}\right)$.

Proof. This is clear by the proof of Lemma D (i).

Lemma $\mathrm{F}\left((\mathrm{P} .8)\right.$, [2]). If $H=\operatorname{ann}\left(X, \mathrm{gp} \cup_{-\infty}^{\infty} \gamma^{j} \Theta\right)$, then there exist subgroups $F^{-}$and $F^{+}$of $F$ satisfying the conditions;

(i) $\sigma H=H$ and the topological entropy of $\sigma_{H}$ equals zero,

(ii) $H$ contains a sequence $H=H_{0} \supset H_{1} \supset \cdots \supset \cap H_{n}=\{0\}$ of subgroups such that for $n \geqslant 0, \sigma H_{n}=H_{n}$ and $H / H_{n}$ is finite,

(iii) $F^{-} \supset \sigma^{-1} F^{-} \supset \cdots \supset \bigcap_{0}^{\infty} \sigma^{-n} F^{-}=\{0\}$,

(iv) $F^{+} \supset \sigma F^{+} \supset \cdots \supset \bigcap_{0}^{\infty} \sigma^{n} F^{+}=\{0\}$,

(v) $\sigma F^{-} / F^{-}$and $F^{+} / \sigma F^{+}$are finite,

(vi) $F=F^{-} \oplus F^{+} \oplus H$.

By Lemmas C (iv) and F (vi), we have $X=\psi\left(\boldsymbol{R}^{r}\right)+\left\{F^{-} \oplus F^{+} \oplus H\right\}$. Since $X$ is connected, it follows that $X=\psi\left(\boldsymbol{R}^{r}\right)+\left\{\boldsymbol{F}^{-} \oplus \boldsymbol{F}^{+}\right\}$when $H$ is finite.

Lemma $G((\mathrm{P} .4),[2]) . \quad$ (i) $H=\{0\}$ iff $G=\mathrm{gp} \cup_{-\infty}^{\infty} \gamma^{j} \Theta$, (ii) $H$ is finite iff $(G, \gamma)$ is finitely generated under $\gamma$; i.e. there is a finite set $\Lambda$ in $G$ such that $G=\operatorname{gp} \bigcup_{-\infty}^{\infty} \gamma^{j} \Lambda$.

The space $R^{r}$ splits into a direct sum $R^{r}=E^{u} \oplus E^{s} \oplus E^{c}$ of $\gamma$-invariant 
subspaces $E^{u}, E^{s}$ and $E^{c}$ such that the eigenvalues of $\gamma_{E^{u}}, \gamma_{E^{s}}$ and $\gamma_{E^{c}}$ have modulus $>1,<1$ and 1 respectively. We call that $\left(\boldsymbol{R}^{r}, \gamma\right)$ is hyperbolic iff $E^{c}=\{0\}$. It is easily proved that there are norms $\|\cdot\|_{u}$ and $\|\cdot\|_{s}$ of $E^{u}$ and $E^{s}$, respectively and $\lambda_{0} \in(0,1)$ such that $\left\|\gamma^{n} x\right\|_{u} \leqslant \lambda_{0}^{-n}\|x\|_{u}\left(x \in E^{u}\right.$ and $n \leqslant 0)$ and $\left\|\gamma^{n} x\right\|_{s} \leqslant \lambda_{0}^{n}\|x\|_{s}\left(x \in E^{s}\right.$ and $\left.n \geqslant 0\right)$. If $E^{c} \neq\{0\}$, by using Jordan normal form in the real field for $\left(E^{c}, \gamma\right)$ we get a finite direct sum $E^{c}=E^{c_{0}} \oplus \cdots \oplus E^{c_{k}}$ of the subspaces $E^{c_{i}}$ satisfying the following conditions; for $0 \leqslant i \leqslant k$, the dimension of $E^{c_{i}}$ is 1 or 2 , and

$$
\gamma_{E^{c}}=\left[\begin{array}{llll}
\gamma_{0} & I_{1} & & \\
& \cdot & & \\
& \gamma_{1} \cdot & I_{k} \\
0 & & \cdot & I_{k} \\
& & & \gamma_{k}
\end{array}\right]
$$

where $r_{i}: E^{c_{i}} \longleftrightarrow$ is an isometry under some norm $\|\cdot\|_{c_{i}}$ of $E^{c_{i}}$ and each $I_{i}: E^{c_{i}} \rightarrow E^{c_{i-1}}$ is either a zero map or a map corresponding to the identity matrix. We call that $\left(\boldsymbol{R}^{r}, \gamma\right)$ is central spin iff $E^{c} \neq\{0\}$ and each $I_{i}$ is a zero map. If the characteristic polynomial $p(x)$ of $\gamma$ is irreducible over $\boldsymbol{Q}$ and $p(x)$ has roots of modulus one, then $\left(\boldsymbol{R}^{r}, \gamma\right)$ is central spin. Define a norm $\|\cdot\|_{c}$ of $E^{c}$ by $\|x\|_{c}=\max \left\{\left\|x^{i}\right\|_{c_{i}}: 0 \leqslant i \leqslant k\right\}\left(x=x^{0}+\cdots+x^{k} \in \oplus_{0}^{k} E^{c_{i}}\right)$. Then we get easily that $d_{0}(x, y)=\max \left\{\left\|x^{u}-y^{u}\right\|_{u},\left\|x^{s}-y^{s}\right\|_{s},\left\|x^{c}-y^{c}\right\|_{c}\right\}$ is a metric of $\boldsymbol{R}^{r}$ satisfying the following conditions; (i) $d_{0}$ is translation invariant, (ii) there is $\lambda_{0} \in(0,1)$ with

$$
d_{0}\left(\gamma^{n} x, 0\right) \leqslant \begin{cases}\lambda_{0}^{-n} d_{0}(x, 0) & \left(x \in E^{u}, n \leqslant 0\right), \\ \lambda_{0}^{n} d_{0}(x, 0) & \left(x \in E^{s}, n \geqslant 0\right),\end{cases}
$$

and (iii) if $E^{c} \neq\{0\}$ then each of $\gamma_{i}$ (under the above notations) is $d_{0^{-}}$ isometry. We see that there are $\alpha_{1}>0$ such that for every $\alpha \in\left(0, \alpha_{1}\right]$, if $B(\alpha)=\left\{x \in R^{r}: d_{0}(x, 0) \leqslant \alpha\right\}$ then $\psi B(\alpha) \oplus F^{-} \oplus F^{+} \oplus H$ is a closed neighborhood of $X$.

Let $\lambda_{0}$ be as above. Then the functions

$$
\begin{aligned}
& d_{-}(x, y)= \begin{cases}\lambda_{0}^{n} & \text { if } x-y \in \sigma^{-n} F^{-} \text {and } x-y \notin \sigma^{-(n+1)} F^{-} \\
0 & \text { if } x=y\end{cases} \\
& d_{+}(x, y)= \begin{cases}\lambda_{0}^{n} & \text { if } x-y \in \sigma^{n} F^{+} \text {and } x-y \notin \sigma^{n+1} F^{+} \\
0 & \text { if } x=y\end{cases}
\end{aligned}
$$

are metrics generating the original topology of $F^{-}$and $F^{+}$respectively. For $x=x_{0}+x_{1}+x_{2}+x_{3} \in \psi B\left(\alpha_{1}\right) \oplus F^{-} \oplus F^{+} \oplus H$, put 


$$
\rho(x)=\min \left\{\alpha_{1}, \max \left\{d_{0}\left(\psi^{-1} x_{0}, 0\right), d_{-}\left(x_{1}, 0\right), d_{+}\left(x_{2}, 0\right), d\left(x_{3}, 0\right)\right\}\right\} .
$$

Then the metric of $X$ defined by

$$
d(x, y)= \begin{cases}\rho(x-y) & \text { if } x-y \in \psi B\left(\alpha_{1}\right) \oplus F^{-} \oplus F^{+} \oplus H \\ \alpha_{1} & \text { otherwise }\end{cases}
$$

is compatible with the original topology. It follows that for $\varepsilon \in\left(0, \alpha_{1}\right)$, $B(\varepsilon)=B^{u}(\varepsilon) \oplus B^{s}(\varepsilon) \oplus B^{c}(\varepsilon)$ where $B^{u}(\varepsilon)=B(\varepsilon) \cap E^{u}, B^{s}(\varepsilon)=B(\varepsilon) \cap E^{s}$ and $B^{c}(\varepsilon)=B(\varepsilon) \cap E^{c}$.

In proving our results, it is important that closed neighborhoods are chosen to be proper subsets of $X$, so that we take and fix a number $\alpha_{0}$ such that

$$
\text { (*) } 0<\alpha_{0}<\min \left\{\alpha_{1}, \alpha_{2}\right\} \text {. }
$$

Here $\alpha_{2}$ is the number chosen in Lemma E. For $\varepsilon \in\left(0, \alpha_{0}\right]$, a closed neighborhood $W(\varepsilon)=\{x \in X: d(x, 0) \leqslant \varepsilon\}$ is expressed as

$$
(* *) \quad W(\varepsilon)=W^{u}(\varepsilon) \oplus W^{s}(\varepsilon) \oplus W^{c}(\varepsilon)
$$

where $W^{u}(\varepsilon)=W(\varepsilon) \cap\left\{\psi B^{u}(\varepsilon) \oplus F^{-}\right\}, \quad W^{s}(\varepsilon)=W(\varepsilon) \cap\left\{\psi B^{s}(\varepsilon) \oplus F^{+}\right\} \quad$ and $W^{c}(\varepsilon)=W(\varepsilon) \cap\left\{\psi B^{c}(\varepsilon) \oplus H\right\}$. Let $d$ be a metric of $X$ defined as above. For $x=x^{u}+x^{s}+x^{c} \in W^{u}\left(\alpha_{0}\right) \oplus W^{s}\left(\alpha_{0}\right) \oplus W^{c}\left(\alpha_{0}\right)$, we have

$$
\begin{array}{r}
d(x, 0)=\max \left\{d\left(x^{u}, 0\right), d\left(x^{s}, 0\right), d\left(x^{c}, 0\right)\right\} \quad \text { and } \\
(* * *) \quad d\left(\sigma^{n} x, 0\right) \leqslant \begin{cases}\lambda_{0}^{-n} d(x, 0) & \left(x \in W^{u}\left(\alpha_{0}\right), n \leqslant 0\right) \\
\lambda_{0}^{n} d(x, 0) & \left(x \in W^{s}\left(\alpha_{0}\right), n \geqslant 0\right) .\end{cases}
\end{array}
$$

Lemma H. If $(X, \sigma)$ is ergodic under the Haar measure, then $\psi\left(E^{c}\right)$ is dense in $X$.

Proof. There is in $E^{c}$ a $\gamma$-invariant subspace $E^{c_{1}}$ such that $\gamma_{E c_{1}}$ is $d_{0^{-}}$ isometry. Hence $E^{c}$ is expressed as $E^{c}=E^{c_{1}} \oplus E^{c_{2}}$ where $E^{c_{2}}$ is a subspace. Assume that $\psi\left(E^{c}\right)$ is not dense in $X$, and put $A=\overline{\psi\left(E^{c_{1}}\right)}$. Then $A$ is a $\sigma$-invariant connected subgroup of $X$. Obviously, $\sigma_{A}$ is $d$-isometry; i.e. the topological entropy of $\sigma_{A}$ is zero (ent $\left(\sigma_{A}\right)=0$ ). As before let $(G, \gamma)$ be the dual of $(X, \sigma)$ and $G_{A}$ be the annihilator of $A$ in $G$. Since $G_{A} \subset$ $G \subset \boldsymbol{R}^{r}$, we denote by $V_{A}$ the smallest vector subgroup of $\boldsymbol{R}^{r}$ containing $G_{A^{*}}$. Then $\boldsymbol{R}^{r}$ is expressed as $\boldsymbol{R}^{r}=V_{A} \oplus V^{\prime}$ where $V^{\prime}$ is a subspace. We see that $\boldsymbol{R}^{r} / V_{A} \cong V^{\prime}$ is the smallest subspace containing $\dot{G}_{A}=G / G_{A}$. Since $G$ is finitely generated under $\gamma$, so is $\dot{G}_{A}$. Let $p(x)$ be the characteristic 
polynomial of $\gamma_{\dot{G}_{A}}$. Then the Kolmogorov entropy of $\sigma_{A}$ equals $h\left(\sigma_{A}\right)=$ $\sum_{|\lambda|>1} \log |\lambda|+\log \Delta$ where $\lambda$ 's are the eigenvalues of $\gamma_{\dot{G}_{A}}$ and $\Delta$ is the smallest positive integer such that $\Delta p(x)$ has the integer coefficients (see [13]). Since ent $\left(\sigma_{A}\right)=h\left(\sigma_{A}\right)=0$, we have $\Delta=1$ (hence $p(x) \in Z[x]$ ) and all the roots of $p(x)$ are modulus one. It follows from a result in the number theory that they are the roots of unity since $p(x) \in Z[x]$. On the other hand, since $(X, \sigma)$ is ergodic, all the roots of the characteristic polynomial of $\gamma$ are not the roots of unity. This is a contradiction.

Remark 1. Let $M$ be a compact manifold and $\varphi$ be a diffeomorphism of $M$. It is proved in [10] that the set of all periodic points is dense in the non-wandering set when $(M, \varphi)$ is topologically stable. In general this is not true for homeomorphisms on compact metric spaces. For example, let $\gamma$ be an automorphism of $\boldsymbol{Q}^{r}$. Consider to $\boldsymbol{Q}^{r}$ be an abelian group imposed with the discrete topology. If $\left(\boldsymbol{R}^{r}, \gamma\right)$ is hyperbolic, then $\left(\gamma^{j}-I\right) \boldsymbol{Q}^{r}=\boldsymbol{Q}^{r}$ for every $j>0$. From this we get that the dual $(X, \sigma)$ of $\left(\boldsymbol{Q}^{r}, \gamma\right)$ has no periodic points except 0 ; i.e. $(X, \sigma)$ is not densely periodic. By Theorem 2 in the next section, $(X, \sigma)$ has the P.O.T.P., and $(X, \sigma)$ is topologically stable by the first statement of Theorem 1 .

Since $\boldsymbol{Q}^{r}$ is not finitely generated under $\gamma,(X, \sigma)$ is not expansive (by Theorem 1, [2]). Therefore it will follow that there is a solenoidal automorphism which has the P.O.T.P., but is not expansive.

Remark 2. The set $\mathscr{C}(X)$ of all non-empty closed subsets of $X$ is a compact metric space by the Hausdorff metric $\bar{d}$. Denote by $\widetilde{O r b}^{\varepsilon}(\sigma)$ the set of all $A \in \mathscr{C}(X)$ for which there is $\left\{x_{i}\right\} \in \operatorname{Orb}^{\circ}(\sigma)$ such that $A=\overline{\left\{x_{i}: i \in Z\right.}$. Let $E(\sigma)$ denote the set of all $A \in \mathscr{C}(X)$ such that for every $\varepsilon>0$ there is $A_{\varepsilon} \in \widetilde{\mathrm{Orb}^{\varepsilon}}(\sigma)$ with $\bar{d}\left(A, A_{\varepsilon}\right)<\varepsilon$. Then $E(\sigma)$ is closed in $\mathscr{C}(X)$. We define

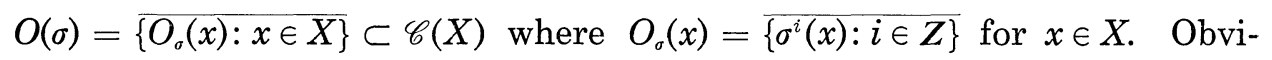
ously $O(\sigma) \subset E(\sigma)$. We call $\sigma$ to have the OE-property iff $O(\sigma)=E(\sigma)$. A. Morimoto asks in [11] whether the lifting system $\left(\boldsymbol{R}^{r}, \gamma\right)$ of $(X, \sigma)$ is hyperbolic if $\sigma$ has the OE-property. In [3] it is proved that for every automorphism $\beta$ of a compact metric group, $\beta$ has the OE-property iff $\beta$ has the P.O.T.P.. From this result together with Theorem 2 in the next section, we shall see that Morimoto's problem is completely solved.

From Theorem 1 and Remark 2 we get the following

Corollary. The following $(\mathrm{A})^{\prime},(\mathrm{B})^{\prime}$ and $(\mathrm{C})^{\prime}$ are equivalent; 
(A) $(X, \sigma)$ is topologically stable,

$(\mathrm{B})^{\prime}(X, \sigma)$ has the P.O.T.P.,

$(\mathrm{C})^{\prime}(X, \sigma)$ satisfies the OE-property.

Hereafter, the restriction and the factor of $\sigma$ will be denoted by the same symbol if there is no confusion.

\section{§2. An auxiliary result}

In this section we shall prove the following

TheOREm 2. The lifting system $\left(\boldsymbol{R}^{r}, \gamma\right)$ of $(X, \sigma)$ is hyperbolic iff $(X, \sigma)$ has the P.O.T.P..

For the proof we need the following lemmas.

Lemma 2.1. $(X, \sigma)$ has the P.O.T.P., then $(X, \sigma)$ is topologically mixing.

Proof. By (Theorem 2, [1]), $X$ contains $\sigma$-invariant subgroups $X_{1}$ and $X_{2}$ such that $\left(X_{1}, \sigma\right)$ has zero entropy, $\left(X_{2}, \sigma\right)$ is ergodic and $X$ splits into a sum $X=X_{1}+X_{2}$. Since $X / X_{2}$ is a factor group of $X_{1},\left(X / X_{2}, \sigma\right)$ has zero entropy. As we saw in the proof of Lemma $\mathrm{H}, X / X_{2}$ is a torus and $\left(X / X_{2}\right.$, $\sigma)$ is not hyperbolic. It is easy to see that $\left(X / X_{2}, \sigma\right)$ has the P.O.T.P., so that we must have $X_{1}=\{0\}$ by Theorem A. Therefore $(X, \sigma)$ is ergodic and hence $(X, \sigma)$ is topologically mixing.

Lemma 2.2. If $(X, \sigma)$ has the P.O.T.P., then $(X, \sigma)$ satisfies weak specification.

Proof. By Lemma 2.1, $(X, \sigma)$ is topologically mixing. Let $\varepsilon>0$ be given. Choose $\delta=\delta(\varepsilon)>0$ as in the definition of the P.O.T.P. Cover $X$ by a finite family $\mathscr{U}$ of $\delta$-balls. For any two $U_{i}, U_{j} \in \mathscr{U}$ there is $M_{i j}>0$ such that $\sigma^{n} U_{i} \cap U_{j} \neq \phi$ for $n \geqslant M_{i j}$. Put $M=\max \left\{M_{i j}: i, j\right\}<\infty$. Let $x_{1}, \cdots, x_{k}$ be points in $X$ and $a_{1} \leqslant b_{1}<\cdots<a_{k} \leqslant b_{k}$ be integers with $a_{j}$ $-b_{j-1} \geqslant M$ for $2 \leqslant j \leqslant k$. For $z \in X$ we denote by $U(z)$ some $U \in \mathscr{U}$ with $z \in U$. For $1 \leqslant j \leqslant k$ there is a point $y_{j} \in U\left(\sigma^{b_{j}} x_{j}\right)$ such that $\sigma^{a_{j+1}-b_{j}} y_{j} \in$ $U\left(\sigma^{a_{j+1}} x_{j+1}\right)$. Consider the $\delta$-pseudo-orbit $\left\{z_{i}: a_{1} \leqslant i \leqslant a_{k}\right\}$ defined by $z_{i}=$ $\sigma^{i} x_{j}$ for $a_{j} \leqslant i \leqslant b_{j}$ and $z_{i}=\sigma^{i-b_{j}}\left(y_{j}\right)$ for $b_{j}<i<a_{j+1}$. Then there is a point $x \in X$ which $\varepsilon$-traces the orbit. From this we get $d\left(\sigma^{i}(x), \sigma^{i}\left(x_{j}\right)\right)<\varepsilon$ for $a_{j} \leqslant i \leqslant b_{j}(1 \leqslant j \leqslant k)$.

LEMma 2.3. Let $\sigma$ be an automorphism of a compact metric group $Y$ 
and $K$ be a completely $\sigma$-invariant normal subgroup of $Y(\sigma(K)=K)$. If both $(Y \mid K, \sigma)$ and $(K, \sigma)$ have the P.O.T.P., then so is $(Y, \sigma)$.

Proof. By assumption, for every $\varepsilon>0$ there is a $\delta>0$ with $\delta<\varepsilon$ such that for every $\delta$-pseudo-orbit in $K$, a point in $K \varepsilon / 2$-traces the orbit. Choose $\eta$ with $0<\eta<\delta / 3$ such that the following conditions hold;

(a) $d(\sigma(x), \sigma(y))<\delta / 3$ when $d(x, y)<\eta$ and

(b) for an arbitrary $\eta$-pseudo-orbit $\left\{x_{i}: a<i<b\right\}$ of $Y, Y \mid K$ contains a point $x+K \in Y / K$ with $d\left(\sigma^{i}(x+K), x_{i}+K\right)<\delta / 3(a<i<b)$ (here $d$ is a metric on $X / K$ defined by $d(x+K, y+K)=\inf \left\{d\left(x+k, y+k^{\prime}\right): k, k^{\prime} \in\right.$ $K\})$.

By (b), for $a<i<b$ there is $k_{i} \in K$ such that $d\left(\sigma^{i}(x)+k_{i}, x_{i}\right)<\eta$.

By (a), $d\left(\sigma^{i+1}(x)+\sigma\left(k_{i}\right), \sigma\left(x_{1}\right)\right)<\delta / 3$. We calculate that for $a<i<b-1$

$$
\begin{aligned}
d\left(\sigma\left(k_{i}\right), k_{i+1}\right) & =d\left(\sigma^{i+1}(x)+\sigma\left(k_{i}\right), \sigma^{i+1}(x)+k_{i+1}\right) \\
& \leqslant d\left(\sigma^{i+1}(x)+\sigma\left(k_{i}\right), \sigma\left(x_{i}\right)\right)+d\left(\sigma\left(x_{i}\right), x_{i+1}\right)+d\left(x_{i+1}, \sigma^{i+1}(x)+k_{i+1}\right) \\
& <\delta,
\end{aligned}
$$

from which there is a point $k \in K \varepsilon / 2$-tracing the orbit $\left\{k_{i}: a<i<b\right\}$. Since

$$
d\left(\sigma^{i}(x+k), x_{i}\right) \leqslant d\left(\sigma^{i}(x+k), \sigma^{i}(x)+k_{i}\right)+d\left(\sigma^{i}(x)+k_{i}, x_{i}\right)<\varepsilon,
$$

the point $x+k \varepsilon$-traces the orbit $\left\{x_{i}: a<i<b\right\}$ in $Y$ and the proof is completed.

Lemma 2.4. Let $Y$ and $\sigma$ be as in Lemma 2.3. If $Y$ contains a sequence $Y=K_{0} \supset K_{1} \supset \cdots \supset \cap K_{n}=\{e\}$ of normal subgroups such that for $n \geqslant 0, \sigma K_{n}=K_{n}$ and $Y / K_{n}$ is finite, then $(Y, \sigma)$ has the P.O.T.P.

Proof. For every $\varepsilon>0$, there are $n>0$ and $\delta$ with $0<\delta<\varepsilon$ such that

$$
\{x \in Y: d(x, 0)<\delta\} \subset K_{n} \subset\{x \in Y: d(x, 0)<\varepsilon\} .
$$

Let $\left\{x_{i}: a<i<b\right\}$ be an arbitrary $\delta$-pseudo-orbit in $Y$; i.e. $d\left(\sigma x_{i}, x_{i+1}\right)<\delta$, $a<i<b-1$ (without loss of generality we may assume $a+1 \leqslant 0$ ). Then $\sigma x_{i}-x_{i+1} \in K_{n}(a<i<b-1)$. Hence $\sigma^{i} x_{0}-x_{i} \in K_{n}$ since $\sigma K_{n}=K_{n}$; i.e. $d\left(\sigma^{i} x_{0}, x_{i}\right)(a<i<b)$. This shows that (Y, $\sigma$ has the P.O.T.P.)

\section{Proof of Theorem 2.}

As we saw in Section $1, \boldsymbol{R}^{r}$ splits into a direct sum $\boldsymbol{R}^{r}=E^{u} \oplus E^{s} \oplus E^{c}$ of the $\gamma$-invariant subspaces. 
Proof of $\Leftarrow)$ : By Lemma 2.2, $(X, \sigma)$ obeys weak specification. Hence $\left(\boldsymbol{R}^{r}, \gamma\right)$ is central spin by (Theorem 2, [2]); i.e. if $E^{c} \neq\{0\}$ then $\gamma$ on $E^{c}$ is $d_{0}$-isometry (the metric $d_{0}$ is defined as in Section 1 ). We shall now prove that if $E^{c} \neq\{0\}$ then $(X, \sigma)$ has not the P.O.T.P.. To do this, assume that $(X, \sigma)$ has the P.O.T.P.; i.e. for every $\varepsilon \in\left(0, \alpha_{0}\right)$ there is $\delta>0$ with $\delta<\varepsilon$ such that every $\delta$-pseudo-orbit is $\varepsilon / 2$-traced by some point of $X$. Fix $0 \neq$ $v_{0} \in E^{c}$ with $d\left(v_{0}, 0\right)<\delta$ and set $z_{j}=j r^{j}\left(v_{0}\right)$ for $j \in Z$. Then it follows that

$$
d\left(\gamma\left(z_{j}\right), z_{j+1}\right)=d_{0}\left(j \gamma^{j+1}\left(v_{0}\right),(j+1) \gamma^{j+1}\left(v_{0}\right)\right)=d_{0}\left(0, v_{0}\right)<\delta,
$$

and so $\left\{z_{j}\right\} \in \operatorname{Orb}^{\delta}(\gamma)$. Put $V_{\varepsilon}(v)=\left\{u \in \boldsymbol{R}^{r}: d_{0}(v, u)<\varepsilon\right\}$ for $v \in \boldsymbol{R}^{r}$. Then there is $k>0$ such that $V_{\varepsilon}\left(k v_{0}\right) \cap V_{\varepsilon}(0)=\phi$. From the relation between the metrics $d_{0}$ and $d$, we have

$$
\delta>d_{0}\left(\gamma\left(z_{j}\right), z_{j+1}\right)=d\left(\sigma \psi\left(z_{j}\right), \psi\left(z_{j+1}\right)\right)
$$

for $j \in Z$, and so $\left\{\psi\left(z_{j}\right)\right\} \in \operatorname{Orb}^{\delta}(\sigma)$. By the assumption there is $x \in X$ such that $d\left(\sigma^{j}(x), \psi\left(z_{j}\right)\right)<\varepsilon / 2$ for $j \in Z$. Since $\psi\left(E^{c}\right)$ is dense in $X$ (by Lemma $\mathrm{H})$, we can find $0 \neq y \in \psi\left(E^{c}\right)$ with $\max \left\{d\left(\sigma^{i}(y), \sigma^{i}(x)\right): 0 \leqslant i \leqslant k\right\} \leqslant \varepsilon / 2$. Hence $d\left(\sigma^{j}(y), \psi\left(z_{j}\right)\right)<\varepsilon$ for $0 \leqslant j \leqslant k$, from which we have

$$
\begin{aligned}
& d\left(y, \psi\left(z_{0}\right)\right)=d(y, 0)=d_{0}\left(\psi^{-1}(y), 0\right)<\varepsilon \text { and } \\
& \begin{aligned}
d\left(\sigma^{k}(y), \psi\left(z_{k}\right)\right) & =d_{0}\left(\gamma^{k} \psi^{-1}(y), z_{k}\right)=d_{0}\left(\gamma^{k} \psi^{-1}(y), \gamma^{k}\left(k v_{0}\right)\right) \\
& =d_{0}\left(\psi^{-1}(y), k v_{0}\right)<\varepsilon .
\end{aligned}
\end{aligned}
$$

Therefore $\psi^{-1}(y) \in V_{\varepsilon}(0) \cap V_{\varepsilon}\left(k v_{0}\right)$, which is a contradiction.

Proof of $\Rightarrow$ ). Since $H$ is the annihilator of $g p \bigcup_{-\infty}^{\infty} \gamma^{n} \Theta$ in $X$ (see Lemma F), $\left(\mathrm{gp} \cup_{-\infty}^{\infty} \gamma^{n} \Theta, \gamma\right)$ is the dual of $(X / H, \sigma)$ and $G / g p \bigcup_{-\infty}^{\infty} \gamma^{n} \Theta$ is a torsion group. Hence the dimension of $\dot{X}=X / H$ is equal to that of $X$ since $H$ is zero-dimensional. Since gp $\bigcup_{-\infty}^{\infty} \gamma^{n} \Theta$ is clearly finitely generated under $\gamma$ and since the lifting system $\left(\boldsymbol{R}^{r}, \gamma\right)$ of $(\dot{\boldsymbol{X}}, \sigma)$ is hyperbolic, it follows that $(\dot{X}, \sigma)$ is expansive (see Theorem 1, [2]). We see that $\dot{X}$ is expressed as $\dot{X}=\psi\left(\boldsymbol{R}^{r}\right)+\left\{F^{-} \oplus F^{+}\right\}$by Lemma $G$ (ii). Let $\varepsilon_{0}>0$ be an expansive constant for $(\dot{X}, \sigma)$. Then, for $0<\varepsilon<\varepsilon_{0}$ we have a coordinate neighborhood $W(\varepsilon)=W^{u}(\varepsilon) \oplus W^{s}(\varepsilon)$ of 0 in $\dot{X}$ where $W^{u}(\varepsilon) \neq\{0\}$ and $W^{s}(\varepsilon)$ $\neq\{0\}$. It is easily seen that there is $\delta=\delta(\varepsilon)>0$ such that if $d(x, y)<$ $\delta(x, y \in \dot{X})$ then $\left\{W^{u}(\varepsilon)+x\right\} \cap\left\{W^{s}(\varepsilon)+y\right\}$ consists only of one point. Therefore $(\dot{X}, \sigma)$ has the P.O.T.P. using $(* * *)$ (for the proof, see p. $74, \mathrm{R}$. Bowen [4]). From this fact together with Lemmas 2.3 and 2.4 and Lemma F (ii), we get the conclusion. 


\section{§ 3. Proof of Theorem 1}

To see the statement $(A) \Rightarrow(B)$, we shall prepare the following three lemmas.

LEmma 3.1. If $(X, \sigma)$ is topologically stable, then $X \backslash$ Fix $(\sigma)$ is dense in $X$ where Fix $(\sigma)=\{x \in X: \sigma(x)=x\}$.

Proof. Notice that Fix ( $\sigma)$ is a subgroup of $X$. Assume that $X \backslash$ Fix $(\sigma)$ is not dense in $X$. Then Fix $(\sigma)$ is open in $X$. Since $X$ is connected, we get $X=$ Fix $(\sigma)$; i.e. $\sigma=$ id. Take $\varepsilon>0$ with $2 \varepsilon<\operatorname{diameter}(X)$ and let $\delta>0$ be as in the definition of topological stability. Now we can find $a \in X$ with $d(a, 0)<\delta$ such that $\{n a: n \in Z\}$ is dense in $X$ (see [5]). Let $f_{a}: X \longleftrightarrow$ be a homeomorphism defined by $f_{a}(x)=x+a(x \in X)$, then $d\left(f_{a}(x), x\right)=d\left(f_{a}(x), \sigma(x)\right)<\delta$. Hence there is a continuous map $h: X \longleftrightarrow$ with $h \circ f_{a}=\sigma \circ h$ and $d(h(x), x)<\varepsilon(x \in X)$. Since $h(a n)=h\left(f_{a}^{n}(0)\right)=\sigma^{n} h(0)$ $=h(0)$ for all $n$, we get $h(x)=h(0)$ for all $x \in X$, and so $\varepsilon>d(h(0), x)$ for $x \in X$. On the other hand, since $\varepsilon<\operatorname{diameter}(X) / 2$, there is $y \in X$ with $d(h(0), y)>\varepsilon$, which is a contradiction.

LEMma 3.2 ([10]). Let $\varphi$ be a homeomorphism of a connected metric space $Y$. Assume that $\varphi$ is uniformly continuous and $Y \backslash$ Fix $(\varphi)$ is dense in $Y$. Take and fix a constant $\delta_{1}>0$ and an integer $k>0$. Then for every $\left\{x_{i}\right\} \in \operatorname{Orb}^{\delta_{1}}(\varphi)$ and $\varepsilon_{1}>0$, there is $\left\{x_{\imath}^{\prime}\right\} \in \operatorname{Orb}^{3 \delta_{1}}(\varphi)$ such that $\left.\mathrm{i}\right) d\left(x_{i}, x_{i}^{\prime}\right)$ $<\varepsilon_{1}$ for $0 \leqslant i \leqslant k$ and ii) $Y_{i}=\left\{\varphi\left(x_{i}^{\prime}\right), x_{i+1}^{\prime}\right\}(0 \leqslant i \leqslant k-1)$ are disjoint.

We shall describe here a proof given in [10] for completeness. We can assume $\varepsilon_{1}<\delta_{1}$. For this $\varepsilon_{1}$, there is $\varepsilon_{1}^{\prime}>0$ with $\varepsilon_{1}>\varepsilon_{1}^{\prime}$ such that $d(x, y)<\varepsilon_{1}^{\prime}$ implies $d(\varphi(x), \varphi(y))<\varepsilon_{1}$. First we can find $x_{\imath}^{\prime} \in Y(0 \leqslant i \leqslant k)$ such that $x_{i}^{\prime} \neq x_{j}^{\prime}(i \neq j)$ and $d\left(x_{i}, x_{i}^{\prime}\right)<\varepsilon_{1}^{\prime}(0 \leqslant i \leqslant k)$. Next we shall show by induction that $Y_{0}, \cdots, Y_{k-1}$ are disjoint by taking $x_{i}^{\prime}$ suitably. Assume that $Y_{i}=\left\{\varphi\left(x_{i}^{\prime}\right), x_{i+1}^{\prime}\right\}(0 \leqslant i \leqslant k-2)$ are disjoint. We shall show that, by changing $x_{k-1}^{\prime}$ and $x_{k}^{\prime}$ if necessary, $Y_{i} \cap Y_{j}=\phi(0 \leqslant i \neq j \leqslant k-1)$. Consider the point $\varphi\left(x_{k-1}^{\prime}\right)$ and assume $\varphi\left(x_{k-1}^{\prime}\right) \in \bigcup_{i=0}^{k-2} Y_{i}$. Then there is a unique $i \leqslant k-1$ such that $\varphi\left(x_{k-1}^{\prime}\right)=x_{i}^{\prime}$ since $x_{k-1}^{\prime} \neq x_{j}^{\prime}(j \leqslant k-2)$ implies $\varphi\left(x_{k-1}^{\prime}\right) \neq \varphi\left(x_{j}^{\prime}\right)$. If $i \leqslant k-2$, we can find $x_{k-1}^{\prime \prime}$ near $x_{k-1}^{\prime}$ such that $\varphi\left(x_{k-1}^{\prime \prime}\right)$ $\neq x_{i}^{\prime}$. If $i=k-1\left(\varphi\left(x_{k-1}^{\prime}\right)=x_{k-1}^{\prime}\right)$, then we can find $x_{k-1}^{\prime \prime}$ near $x_{k-1}^{\prime}$ such that $\varphi\left(x_{k-1}^{\prime \prime}\right) \neq x_{k-1}^{\prime \prime}$, since $Y \backslash$ Fix $(\varphi)$ is dense and open in $Y$. We denote $x_{k-1}^{\prime \prime}$ by $x_{k-1}^{\prime}$ again. Then we can assume that $x_{k}^{\prime} \notin \bigcup_{i=0}^{k=1} Y_{i}$, since $\bigcup_{i=0}^{k=2} Y_{i}$ is a finite set. Thus we have proved that $Y_{0}, Y_{1}, \ldots, Y_{k-1}$ are disjoint. 
For $i<0$ (resp. $i>k$ ) we define $x_{i}^{\prime}=\varphi^{-i}\left(x_{0}^{\prime}\right)\left(\right.$ resp. $\left.x_{i}^{\prime}=\varphi^{i-k}\left(x_{k}^{\prime}\right)\right)$. Then we see that $\left\{x_{i}^{\prime}\right\} \in \operatorname{Orb}^{3 \delta_{1}}(\varphi)$ since

$$
\begin{aligned}
d\left(\varphi\left(x_{i}^{\prime}\right), x_{i+1}^{\prime}\right) & \leqslant d\left(\varphi\left(x_{i}^{\prime}\right), \varphi\left(x_{i}\right)\right)+d\left(\varphi\left(x_{i}\right), x_{i+1}\right)+d\left(x_{i+1}, x_{i+1}^{\prime}\right) \\
& <\varepsilon_{1}+\delta_{1}+\varepsilon_{1}^{\prime}<3 \delta_{1}
\end{aligned}
$$

for $0 \leqslant i \leqslant k-1$. This completes the proof of Lemma 3.2.

Lemma 3.3 ([8]). Let $M$ be a differentiable manifold of $\operatorname{dim}(M) \geqslant 2$ with a metric $d$. Let $M_{i}=\left\{p_{i}, q_{i}\right\}(1 \leqslant i \leqslant k)$ be a subset of $M$ consisting of at most two points $p_{i}$ and $q_{i}$ with $d\left(p_{i}, q_{i}\right)<\delta$. Assume that $M_{i} \cap$ $M_{j}=\phi(i \neq j)$. Then there is an onto homeomorphism $\eta: M \longleftrightarrow$ such that $d(\eta(x), x)<2 \pi \delta$ for $x \in M$, and that $\eta\left(p_{i}\right)=q_{i}(1 \leqslant i \leqslant k)$.

Proof of the statement $(\mathrm{A}) \Rightarrow(\mathrm{B})$. The proof will be done along the following two cases.

Case (1). For the case $\operatorname{dim}(X)=1$, we get that $\left(\boldsymbol{R}^{1}, \gamma\right)$ is hyperbolic by applying Lemma 3.1. Therefore $(X, \sigma)$ has the P.O.T.P. by Theorem 2.

Case (2). We can use Lemma 3.3 when $\operatorname{dim}(X) \geqslant 2$. For every $\varepsilon>$ 0 , choose $\delta>0(\delta<\varepsilon)$ as in the definition of topological stability. Since $\overline{\psi\left(\boldsymbol{R}^{r}\right)}=X$ by Lemma B, for every $\left\{x_{i}\right\} \in \operatorname{Orb}^{\delta / 12 \pi}(\sigma)$ there is $\left\{x_{i}^{\prime}\right\} \subset \psi\left(\boldsymbol{R}^{r}\right)$ such that $d\left(x_{i}, x_{i}^{\prime}\right)<\delta / 24 \pi, d\left(\sigma\left(x_{i}\right), \sigma\left(x_{i}^{\prime}\right)\right)<\delta / 24 \pi$ and $\sigma\left(x_{i}^{\prime}\right)-x_{i+1}^{\prime} \in W(\delta / 6 \pi)(i \in Z)$ where $W(\delta / 6 \pi)$ is a closed neighborhood with the radius $\delta / 6 \pi$ of 0 in $X$. Since $(X, \sigma)$ is topologically stable and $\psi\left(\boldsymbol{R}^{r}\right)$ is connected, we have that $\psi\left(\boldsymbol{R}^{r}\right) \backslash\left(\psi\left(\boldsymbol{R}^{r}\right) \cap\right.$ Fix $\left.(\sigma)\right)$ is dense in $\psi\left(\boldsymbol{R}^{r}\right)$ (by using Lemma 3.1).

Take and fix an integer $k>0$. Notice that $\sigma$ and $\sigma^{-1}$ are uniformly continuous on $\psi\left(\boldsymbol{R}^{r}\right)$. By Lemma 3.2 there is a sequence $\left\{x_{i}^{\prime \prime}\right\} \in \operatorname{Orb}^{\delta / 2 \pi}(\sigma)$ such that $d\left(x_{i}^{\prime}, x_{i}^{\prime \prime}\right)<\delta / 2 \pi(0 \leqslant i \leqslant k)$ and $\left\{\sigma\left(x_{i}^{\prime \prime}\right), x_{i+1}^{\prime \prime}\right\}(0 \leqslant i \leqslant k-1)$ are mutually disjoint. Choose two closed balls $B^{\prime}$ and $B$ of 0 in $V_{1} \oplus V_{2}$ such that $\left\{x_{i}^{\prime \prime}\right\}_{1}^{k} \cup\left\{\sigma x_{i}^{\prime \prime}\right\}_{1}^{k-1} \subset \psi^{*}\left(B^{\prime}\right) \subsetneq \psi^{*}(B)$. Since $\psi^{*}(B)$ is a differentiable manifold, as in Lemma 3.3 there is an onto homeomorphism $\eta_{1}: \psi^{*}(B) \longleftrightarrow$ such that $d\left(\eta_{1}(x), x\right)<\delta\left(x \in \psi^{*}(B)\right), \eta_{1}(x)=x$ for $x \in \psi^{*}(B) \backslash \psi^{*}\left(B^{\prime}\right)$ and $\eta_{1} \sigma\left(x_{i}^{\prime \prime}\right)=x_{i+1}^{\prime \prime}(1 \leqslant i \leqslant k-1)$. Choose a small open subgroup $F^{\prime}$ of $F$ such that $\psi^{*}(B+B) \cap F^{\prime}=\{0\}$ and $\psi^{*}(B)+F^{\prime}$ is a closed neighborhood of $X$. Define a map $\eta_{1}^{\prime}: \psi^{*}(B)+F^{\prime} \longleftrightarrow$ by $\eta_{1}^{\prime}(x+y)=\eta_{1}(x)+y\left(x \in \psi^{*}(B)\right.$ and $\left.y \in F^{\prime}\right)$. Then it is clear that $\eta_{1}^{\prime}$ is $1-1$ and onto. Let $\eta$ be a map from $X$ onto itself defined by

$$
\eta(x)= \begin{cases}\eta_{1}^{\prime}(x) & \text { if } x \in \psi^{*}(B)+F^{\prime} \\ x & \text { if } x \notin \psi^{*}(B)+F^{\prime}\end{cases}
$$


It is easily checked that $\eta: X \longleftrightarrow$ is an onto homeomorphism having the properties; $d(\eta(x), x)<\delta$ for $x \in X, \eta(x)=x$ on $\psi^{*}\left(B^{\prime}\right)+F$ and $\eta \sigma\left(x_{i}^{\prime \prime}\right)=$ $x_{i+1}^{\prime \prime}(1 \leqslant i \leqslant k-1)$. Put $\varphi=\eta \circ \sigma$. Since $d(\varphi(x), \sigma(x))<\delta$ for $x \in X$, we have $\left\{\varphi^{i}(x)\right\} \in \operatorname{Orb}^{\delta}(\sigma)$. By the property for $\delta>0$, there is a continuous. map $h: X \longleftrightarrow$ such that $h \circ \varphi=\sigma \circ h$ and $d(h(x), x)<\varepsilon$ for $x \in X$. If $x=$ $h\left(x_{0}^{\prime \prime}\right)$, then for $0 \leqslant i \leqslant k$

$$
\begin{aligned}
d\left(\sigma^{i}(x), x_{i}\right) & =d\left(\sigma^{i}\left(h\left(x_{0}^{\prime \prime}\right)\right), x_{i}\right)=d\left(h\left(\varphi^{i}\left(x_{0}^{\prime \prime}\right), x_{i}\right)\right. \\
& \leqslant d\left(h\left(x_{i}^{\prime \prime}\right), x_{i}^{\prime \prime}\right)+d\left(x_{i}^{\prime \prime}, x_{i}^{\prime}\right)+d\left(x_{i}^{\prime}, x_{i}\right)<2 \varepsilon,
\end{aligned}
$$

which shows $x \in \operatorname{Tr}^{2 \varepsilon}\left(\left\{x_{i}\right\}_{0}^{k}, \sigma\right)$. Since $k$ is arbitrary, $(X, \sigma)$ has the P.O.T.P.

It remains to prove the statement $(B) \Rightarrow(A)$. First we shall prepare the following two lemmas.

Lemma 3.4. Let $\alpha_{0}$ be as in (*). For every $f \in \mathscr{H}(X)$ with $\max \{d(\sigma, f)$, $\left.d\left(\sigma^{-1}, f^{-1}\right)\right\}<\alpha_{0} / 2$, there exist $y_{0} \in F$ and $f_{0} \in \mathscr{H}(X)$ with $f_{0}\left(\psi\left(\boldsymbol{R}^{r}\right)\right)=\psi\left(\boldsymbol{R}^{r}\right)$ such that $f(x)=y_{0}+f_{0}(x)$ for $x \in X$.

Proof. Put $g(x)=f(x)-y_{0}^{\prime}(x \in X)$ where $y_{0}^{\prime}=f(0)$. Since $d(f(0), \sigma(0))$ $=d\left(y_{0}^{\prime}, 0\right)<\alpha_{0} / 2$, we get $d(g(x), f(x))<\alpha_{0} / 2(x \in X)$. Notice that $\{x \in X$ : $\left.d(x, 0)<\alpha_{0}\right\} \subset \psi B\left(\alpha_{0}\right) \oplus F($ see $(* *))$. Put $k(x)=g(x)-\sigma(x)(x \in X)$. Since $d(\kappa(x), 0) \leqslant d(g(x), f(x))+d(f(x), \sigma(x))<\alpha_{0}$ for all $x \in X$, we have $\kappa(x) \epsilon$ $\psi B\left(\alpha_{0}\right) \oplus F$ for $x \in X$ and so $\kappa\left(\psi \boldsymbol{R}^{r}\right) \subset \psi B\left(\alpha_{0}\right) \oplus F$. Since $\psi\left(\boldsymbol{R}^{r}\right)$ contains the identity 0 and $\kappa\left(\psi \boldsymbol{R}^{r}\right)$ is connected, we have $\kappa\left(\psi \boldsymbol{R}^{r}\right) \subset \psi \boldsymbol{B}\left(\alpha_{0}\right)$, from which $g\left(\psi \boldsymbol{R}^{r}\right) \subset \psi\left(\boldsymbol{R}^{r}\right)$. In the same way, it follows that $g^{-1}\left(\psi \boldsymbol{R}^{r}\right) \subset \psi\left(\boldsymbol{R}^{r}\right)$, so that $g\left(\psi \boldsymbol{R}^{r}\right)=\psi\left(\boldsymbol{R}^{r}\right)$. Since $y_{0}^{\prime} \in X=\psi\left(\boldsymbol{R}^{r}\right)+F, y_{0}^{\prime}$ splits into the sum $y_{0}^{\prime}=\psi(v)+y_{0}$ with $\psi(v) \in \psi\left(\boldsymbol{R}^{r}\right)$ and $y_{0} \in F$. Put $f_{0}(x)=\psi(v)+g(x)(x \in X)$. Then $f_{0}(x)$ satisfies all the conditions of the lemma.

LEMMA 3.5 ([10]). Let $Y$ be a metric space such that every bounded set is relatively compact. Let $f: Y \longleftrightarrow$ be a homeomorphism with the P.O.T.P.. If $(Y, f)$ is expansive, then $(Y, f)$ is topologically stable.

We shall give here a proof due to A. Morimoto [10]. The proof will be used in proving the statement $(B) \Rightarrow(A)$. For every $\varepsilon>0$, there is $\delta>0$ such that $\left\{x_{i}\right\} \in \operatorname{Orb}^{\delta}(f)$ implies $\operatorname{Tr}^{\varepsilon}\left(\left\{x_{i}\right\}, f\right)=\phi$. We can assume $\varepsilon<\varepsilon_{0} / 4$, where $\varepsilon_{0}$ is an expansive constant of $f$. Take a $g \in \mathscr{H}(Y)$ with $d(g, f)<\delta$. We shall prove that there exists a continuous map $h: Y \longleftrightarrow$ having the property in the definition of topological stability. Take a point $x \in Y$. It is easy to see that $\left\{g^{i}(x)\right\} \in \operatorname{Orb}^{\delta}(f)$. Hence there is $y \in$ 
$\operatorname{Tr}^{\varepsilon}\left(\left\{g^{i}(x)\right\}, f\right)$; i.e. $d\left(f^{i}(y), g^{i}(x)\right) \leqslant \varepsilon$ for $i \in Z$. If $y^{\prime} \in \operatorname{Tr}^{\varepsilon}\left(\left\{g^{i}(x)\right\}, f\right)$, then we have

$$
d\left(f^{i}(y), f^{i}\left(y^{\prime}\right)\right) \leqslant d\left(f^{i}(y), g^{i}(x)\right)+d\left(g^{i}(x), f^{i}\left(y^{\prime}\right)\right) \leqslant 2 \varepsilon<\varepsilon_{0},
$$

which implies $y=y^{\prime}$. Thus by putting $h(x)=y$, we get a well-defined map $h: Y \longleftrightarrow$ with the property

$$
d\left(f^{i}(h(x)), g^{i}(x)\right) \leqslant \varepsilon \quad \text { for } i \in Z .
$$

Putting $i=0$ in (1) we get

$$
d(h(x), x) \leqslant \varepsilon \quad \text { for } x \in Y .
$$

Next we have, again by (1) for $x$ and $g(x)$,

$$
\begin{aligned}
d\left(f^{i}(f(h(x))), f^{i}(h(g(x)))\right) \leqslant & d\left(f^{i}(f(h(x))), g^{i}(g(x))\right) \\
& +d\left(f^{i}(h(g(x))), g^{i}(g(x))\right) \leqslant 2 \varepsilon<\varepsilon_{0}
\end{aligned}
$$

for every $i \in Z$, which implies $f(h(x))=h(g(x))$. Finally we shall prove the continuity of $h$. Assume that $h$ is not continuous at $x_{0} \in Y$. Then, there is a sequence $x_{\nu} \rightarrow x_{0}(\nu \rightarrow \infty)$ such that $y_{\nu}=h\left(x_{\nu}\right)$ does not tend $y_{0}=h\left(x_{0}\right)$ as $\nu \rightarrow \infty$. Since $\left\{x_{\nu}\right\}$ is bounded and $d\left(h\left(x_{\nu}\right), x_{\nu}\right) \leqslant \varepsilon$ for $\nu>0$, the set $\left\{h\left(x_{\nu}\right)\right\}$ is also bounded. Hence we can assume, by taking a subsequence if necessary, that $y_{\nu} \rightarrow y_{0}^{\prime} \neq y_{0}(\nu \rightarrow \infty)$. Since $f$ is expansive, there is $k \in Z$ such that $d\left(f^{k}\left(y_{0}^{\prime}\right), f^{k}\left(y_{0}\right)\right) \geqslant \varepsilon_{0}$. Fixing $k$, we can find $\nu_{0}>0$ such that for $\nu>\nu_{0}$

$$
d\left(f^{k}\left(y_{\nu}\right), f^{k}\left(y_{0}^{\prime}\right)\right)<\varepsilon_{0} / 4,
$$

since $f^{k}$ is continuous and $y_{\nu} \rightarrow y_{0}^{\prime}(\nu \rightarrow \infty)$. We can assume

$$
d\left(g^{k}\left(x_{\nu}\right), g^{k}\left(x_{0}\right)\right)<\varepsilon \quad\left(\nu \geqslant \nu_{0}\right),
$$

since $g^{k}$ is continuous and $x_{\nu} \rightarrow x_{0}(\nu \rightarrow \infty)$. Now we have, using (2) and (4),

$$
\begin{aligned}
d\left(f^{k}\left(y_{\nu}\right), f^{k}\left(y_{0}\right)\right)= & d\left(f^{k}\left(h\left(x_{\nu}\right)\right), h\left(g^{k}\left(x_{0}\right)\right)\right) \\
= & d\left(h\left(g^{k}\left(x_{\nu}\right)\right), h\left(g^{k}\left(x_{0}\right)\right)\right) \\
\leqslant & d\left(h\left(g^{k}\left(x_{\nu}\right)\right), g^{k}\left(x_{\nu}\right)\right)+d\left(g^{k}\left(x_{\nu}\right), g^{k}\left(x_{0}\right)\right) \\
& +d\left(g^{k}\left(x_{0}\right), h\left(g^{k}\left(x_{0}\right)\right)\right)<3 \varepsilon
\end{aligned}
$$

and hence by (3) we obtain

$$
\begin{aligned}
\varepsilon_{0} & \leqslant d\left(f^{k}\left(y_{0}^{\prime}\right), f^{k}\left(y_{0}\right)\right) \leqslant d\left(f^{k}\left(y_{0}^{\prime}\right), f^{k}\left(y_{\nu}\right)\right)+d\left(f^{k}\left(y_{\nu}\right), f^{k}\left(y_{0}\right)\right) \\
& \leqslant \varepsilon_{0} / 4+3 \varepsilon<\varepsilon_{0},
\end{aligned}
$$


which is a contradiction. This completes the proof of Lemma 3.5.

Proof of the statement $(\mathrm{B}) \Rightarrow(\mathrm{A})$. Remark that $\psi^{*}\left(V_{1} \oplus V_{2}\right)=\psi\left(\boldsymbol{R}^{r}\right)$ (by Lemma D (i)). By Theorem $2,\left(\boldsymbol{R}^{r}, \boldsymbol{\gamma}\right)$ is hyperbolic. Hence $\left(\boldsymbol{R}^{r}, \boldsymbol{\gamma}\right)$, and so $\left(V_{1} \oplus V_{2}, \gamma\right)$, is expansive and has the P.O.T.P. (see Theorem A). Using Lemma 3.5, we get that $\left(V_{1} \oplus V_{2}, \gamma\right)$ is topologically stable; i.e. take $\varepsilon \epsilon$ $\left(0, \alpha_{0} / 2\right)$ such that $\gamma B(3 \varepsilon) \subset B\left(\alpha_{0}\right)$ and $\gamma^{-1} B(3 \varepsilon) \subset B\left(\alpha_{0}\right)$ and let $\delta>0(\delta<\varepsilon)$ be the number with the property of topological stability.

Take $f \in \mathscr{H}(X)$ with $d(f, \sigma)<\delta$ (Then we may assume that the number $\delta$ is chosen such that $\left.d\left(f^{-1}, \sigma^{-1}\right)<\alpha_{0} / 2\right)$. By Lemma 3.4 there are $y_{0} \in F$ and $f_{0} \in \mathscr{H}(X)$ such that $f_{0}\left(\psi\left(\boldsymbol{R}^{r}\right)\right)=\psi\left(\boldsymbol{R}^{r}\right)$ and $f(x)=f_{0}(x)+y_{0}$ for $x \in X$. Since $\overline{\psi\left(Z^{r}\right)}=F$ by Lemma D (iii), we can choose in $\psi\left(Z^{r}\right)$ a sequence $\left\{y_{n}\right\}_{n \geqslant 1}$ such that $y_{n} \rightarrow y_{0}$ as $n \rightarrow \infty$. Put $f_{n}(x)=y_{n}+f_{0}(x)$. Then $d\left(f_{n}, \sigma\right)$ $<\delta$ for $n$ large enough. Fix such an integer $n$ and define $\hat{f}_{n}(v)=\psi^{*-1}$ $f_{n} \psi^{*}(v)\left(v \in V_{1} \oplus V_{2}\right)$. Then we claim that $\hat{f}_{n}: V_{1} \oplus V_{2} \longleftrightarrow$ is uniformly continuous. Indeed, we denote by $F(\varepsilon)$ a closed neighborhood with the radius $\varepsilon$ of 0 in $F$. Since $f_{n}: X \longleftrightarrow$ is uniformly continuous, for every $\lambda \epsilon$ $\left(0, \alpha_{0}\right]$ there is $\alpha>0$ with $\alpha<\lambda$ such that for every $v \in V_{1} \oplus V_{2}$

$$
f_{n}\left(\psi^{*} B(\alpha) \oplus F(\alpha)+\psi^{*}(v)\right) \subset \psi^{*} B(\lambda) \oplus F(\lambda)+f_{n} \psi^{*}(v),
$$

from which

$$
f_{n}\left(\psi^{*} B(\alpha)+\psi^{*}(v)\right) \subset \psi^{*} B(\lambda)+f_{n} \psi^{*}(v) .
$$

Hence, $\hat{f}_{n}(B(\alpha)+v) \subset B(\lambda)+\hat{f}_{n}(v)$; i.e. our requirement was obtained.

Let $d_{0}$ be a metric of $V_{1} \oplus V_{2}$ defined as in Section 1 . Since $r=$ $\psi^{-1} \circ \sigma \circ \psi$, we have

$$
\delta>d\left(\sigma \psi^{*}(v), f_{n} \psi^{*}(v)\right)=d_{0}\left(\psi^{*-1} \sigma \psi^{*}(v), \psi^{*-1} f_{n} \psi^{*}(v)\right)=d_{0}\left(\gamma(v), \hat{f}_{n}(v)\right),
$$

so that $\left\{\hat{f}_{n}(v)\right\} \in \operatorname{Orb}^{\delta}(\gamma)$ for $v \in V_{1} \oplus V_{2}$. Hence there is $w \in V_{1} \oplus V_{2}$ with $d_{0}\left(\hat{f}_{n}^{j}(v), \gamma^{j}(w)\right)<\varepsilon(j \in Z)$ since $\left(V_{1} \oplus V_{2}, \gamma\right)$ has the P.O.T.P.. Put $w=\hat{h}_{n}(v)$. Since $\left(V_{1} \oplus V_{2}, \gamma\right)$ is expansive, from the proof of Lemma 3.5 it follows that $\hat{h}_{n}: V_{1} \oplus V_{2} \longleftrightarrow$ is a continuous map such that $\hat{h}_{n} \circ \hat{f}_{n}=\alpha \circ \hat{h}_{n}$ and $d_{0}\left(\hat{h}_{n}, \mathrm{id}\right)<\varepsilon$. Put $h_{n}=\psi^{*} \circ \hat{h}_{n} \circ \psi^{*-1}$. Obviously, $h_{n} \circ f_{n}=\sigma \circ h_{n}$ on $\psi\left(\boldsymbol{R}^{r}\right)$ and $d\left(h_{n}(x), x\right)<\varepsilon$ for $x \in \psi\left(\boldsymbol{R}^{r}\right)$.

We now prove that $h_{n}$ is uniformly continuous. Since $d\left(\hat{f}_{n}^{j}(v), \gamma^{j} \hat{h}_{n}(v)\right)$ $<\varepsilon$ for $j \in Z$ and $v \in V_{1} \oplus V_{2}$, we have

$$
\psi^{*-1} f_{n}^{j} \psi^{*}(v)-\gamma^{j} \hat{h}_{n}(v)=\hat{f}_{n}^{j}(v)-\gamma^{j} \hat{h}_{n}(v) \in B(\varepsilon),
$$


so that for all $v \in V_{1} \oplus V_{2}$ and all $j \in Z$

$$
f_{n}^{j} \psi^{*}(v)-\sigma^{j} h_{n} \psi^{*}(v) \in \psi^{*} B(\varepsilon) .
$$

Since $\left(\boldsymbol{R}^{r}, \gamma\right)$ is expansive, it is easily checked that for every $\lambda>0$ $(\lambda<\varepsilon)$ there is $N \geqslant 1$ such that $d\left(h_{n}(x), h_{n}(y)\right)<\lambda$ when $\sigma^{j} h_{n}(x)-\sigma^{j} h_{n}(y)$ $\in \psi^{*} B(3 \varepsilon)$ for $j$ with $|j| \leqslant N$. Take $\alpha>0$ such that if $d(x, y)<\alpha$ for $x$, $y \in \psi\left(\boldsymbol{R}^{r}\right)$ then $\max \left\{d\left(f_{n}^{j}(x), f_{n}^{j}(y)\right):-N \leqslant j \leqslant N\right\}<\lambda$. Then for $j$ with $|j| \leqslant N$

$$
\begin{aligned}
d\left(\sigma^{j} h_{n}(x), \sigma^{j} h_{n}(y)\right) \leqslant & d\left(\sigma^{j} h_{n}(x), f_{n}^{j}(x)\right)+d\left(f_{n}^{j}(x), f_{n}^{j}(y)\right) \\
& +d\left(f_{n}^{j}(y), \sigma^{j} h_{n}(y)\right)<2 \varepsilon+\lambda<3 \varepsilon,
\end{aligned}
$$

which shows that $d\left(h_{n}(x), h_{n}(y)\right)<\lambda$. Indeed, fix $y \in \psi\left(\boldsymbol{R}^{r}\right)$ and put $\kappa_{y}(x)$ $=f_{n}^{j}(x)-f_{n}^{j}(y)$. Then $\kappa_{y}(W(\alpha)+y) \subset \psi^{*} B(\lambda) \oplus F(\lambda)$ where $W(\alpha)=\psi^{*} B(\alpha)$ $\oplus \boldsymbol{F}(\alpha)$. Since $\kappa_{y}\left(\psi\left(\boldsymbol{R}^{r}\right)\right)=\psi\left(\boldsymbol{R}^{r}\right)$ and $\psi\left(\boldsymbol{R}^{r}\right)=U \kappa_{y}(W(\alpha)+y)$, we have $\kappa_{y}(x) \in \kappa_{y}(W(\alpha)+y) \subset \psi^{*} B(\lambda)$ and hence $\sigma^{j} h_{n}(x)-\sigma^{j} h_{n}(y) \in \psi^{*} B(3 \varepsilon)$.

Therefore $h_{n}$ is uniformly extended to a continuous map from $X$ into itself. We shall denote it by the same symbol. By using (5) we have for $m$ and $n$ large enough and for all $x \in X$

$$
\sigma^{j}\left\{h_{n}(x)-h_{m}(x)\right\}+\left\{f_{m}^{j}(x)-f_{n}^{j}(x)\right\} \in \psi^{*} B(2 \varepsilon) .
$$

Since for fixed $j$

$$
\lim _{n, m \rightarrow \infty} d\left(f_{n}^{j}, f_{m}^{j}\right)=0,
$$

there is $N(j)>0$ such that $f_{n}^{j}(x)-f_{m}^{j}(x) \in \psi^{*} B(\varepsilon)$ for $n, m \geqslant N(j)$ and $x$ $\in X$. Hence for $n, m \geqslant N(j)$ and $x \in X$

$$
h_{n}(x)-h_{m}(x) \in \sigma^{-j} \psi^{*} B(3 \varepsilon) .
$$

As before we have $B(3 \varepsilon)=B(3 \varepsilon)^{u} \oplus B(3 \varepsilon)^{s}$ where $B(3 \varepsilon)^{u}=B(3 \varepsilon) \cap E^{u}$ and $B(3 \varepsilon)^{s}=B(3 \varepsilon) \cap E^{s}$. Hence $\psi^{*} B(3 \varepsilon)=\psi^{*} B(3 \varepsilon)^{u} \oplus \psi^{*} B(3 \varepsilon)^{s}$. Since $r B(3 \varepsilon) \subset$ $B\left(\alpha_{0}\right)$ and $\gamma^{-1} B(3 \varepsilon) \subset B\left(\alpha_{0}\right)$, obviously $\sigma \psi^{*} B(3 \varepsilon) \subset \psi^{*} B\left(\alpha_{0}\right)$ and $\sigma^{-1} \psi^{*} B(3 \varepsilon)$ $\subset \psi^{*} B\left(\alpha_{0}\right)$. It follows easily that $\sigma \psi^{*} B(3 \varepsilon)^{u} \subset \psi^{*} B\left(\alpha_{0}\right)^{u}$ and $\sigma^{-1} \psi^{*} B(3 \varepsilon)^{s}$ $\subset \psi^{*} B\left(\alpha_{0}\right)^{s}$. Hence $\bigcap_{-\infty}^{\infty} \sigma^{j} \psi^{*} B(3 \varepsilon)=\bigcap_{-\infty}^{\infty} \sigma^{j} \psi^{*} B(3 \varepsilon)^{u} \oplus \bigcap_{-\infty}^{\infty} \sigma^{j} \psi^{*} B(3 \varepsilon)^{s}=$ $\{0\}$. From (6) we have for $n, m \geqslant \max \{N(j):-i \leqslant j \leqslant i\}$ and $x \in X$

$$
h_{n}(x)-h_{m}(x) \in \bigcap_{j=-i}^{i} \sigma^{j} \psi^{*} B(3 \varepsilon) .
$$

For any open neighborhood $U$ of 0 there is $i>0$ such that $\bigcap_{j=-i}^{i} \sigma^{j} \psi^{*} B(3 \varepsilon)$ $\subset U$. This implies that $\lim _{n, m \rightarrow \infty} d\left(h_{n}, h_{m}\right)=0$; i.e. $\left\{h_{n}\right\}$ converges uni- 
formly to some continuous map $h$ of $X$. Since $h_{n} \circ f_{n}=\sigma \circ h_{n}$ on $X$ and $d\left(h_{n}\right.$, id $)<\varepsilon$ for an arbitrary large $n$, it follows that $h \circ f=\sigma \circ h$ on $X$ and $d(h, \mathrm{id}) \leqslant \varepsilon$. Therefore $(X, \sigma)$ is topologically stable. The proof of Theorem 1 is completed.

\section{REFERENCES}

[1] N. Aoki, A group automorphism is a factor of a direct product of a zero entropy and a Bernoulli automorphism, Fund. Math., 114 (1981), 159-171.

[2] N. Aoki, M. Dateyama and M. Komuro, Solenoidal automorphisms with specification, Monatsh. Math., 93 (1982), 79-110.

[ 3 ] N. Aoki and M. Dateyama, The OE-property of group automorphisms, (to appear in J. Math. Soc. Japan, 36).

[4] R. Bowen, Equilibrium states and the ergodic theory of Anosov diffeomorphisms, Lecture Notes in Math., 470, Berlin-Heiderberg-New York: Springer, 1975.

[ 5 ] P. R. Halmos and H. Samelson, On monothetic groups, Proc. Nat. Acad. Sci. U.S.A., 28 (1942), 254-258.

[6] S. Lang, Algebra, Addison-Wesley, 1972.

[ 7 ] A. G. Kurosch, The Theory of Groups I and II, New York, Chelsea, 1960.

[8] Z. Nitecki and M. Shub, Filtrations, decompositions and explosions, Amer. J. Math., 97 (1976), 1029-1047.

[9] A. Morimoto, Stochastically stable diffeomorphisms and Takens's conjecture, (preprint)

[10] - Stochastic stability of group automorphisms, (preprint)

[11] - The method of the pseudo-orbit tracing property and stability, Tokyo Univ. Seminary notes 39, 1979 (Japanese)

[12] L. Pontrjagin, Topological Groups, Godon and Breach Science Publ. Inc., 1966.

[13] S. A. Yuzvinskii, Calculation of the entropy of a group endomorphism, Siberian Math. J., 8 (1967), 230-239.

Tokyo Metropolitan University

Department of Mathematics

Tokyo, Japan 